\title{
Managing depressive symptoms in people with mild cognitive impairment and mild dementia with a multicomponent psychotherapy intervention: a randomized controlled trial
}

\author{
Johanne B. Tonga, ${ }^{1,2,3}$ Jūrate Šaltytė Benth, ${ }^{4,5}$ Espen A. Arnevik, ${ }^{6}$ Katja Werheid, ${ }^{7}$ \\ Maria S. Korsnes, ${ }^{1}$ and Ingun D. Ulstein ${ }^{8}$ \\ ${ }^{1}$ Department of Old Age Psychiatry, Oslo University Hospital, Gaustad, Postbox 4956, Nydalen, 0424 Oslo, Norway \\ ${ }^{2}$ Norwegian Health Association, Postbox 7139 Majorstuen, 0207 Oslo, Norway \\ ${ }^{3}$ Institute of Psychology, University of Oslo, Postbox 1094, Blindern, 0317 Oslo, Norway \\ ${ }^{4}$ Institute of Clinical Medicine, Campus Ahus, University of Oslo, P.O.Box 1171, 0318 Blindern, Norway \\ ${ }^{5}$ Health Services Research Unit, Akershus University Hospital, Postbox 1000, 1478 Lorenskog, Norway \\ ${ }^{6}$ Division of Mental Health and Addiction, Oslo University Hospital, Postbox 4956, Nydalen, 0424 Oslo, Norway \\ ${ }^{7}$ Clinical Neuropsychology, Department of Psychology, Humboldt-University, Berlin, Rudower Chaussee 18, 12489 Berlin, Germany \\ ${ }^{8}$ Department of Geriatric Medicine, Oslo University Hospital, Ullevål, Postbox 4956, Nydalen, 0424 Oslo, Norway
}

Abstract

Objective: To evaluate the feasibility and effectiveness of the CORDIAL program, a psychosocial intervention consisting of cognitive behavioral therapy (CBT), cognitive rehabilitation, and reminiscence to manage depressive symptoms for people with mild cognitive impairment (MCI) or dementia.

Design: We conducted a randomized controlled trial, based on a two-group (intervention and control), pre-/post-intervention design.

Setting: Participants were recruited from five different old age psychiatry and memory clinics at outpatients' hospitals.

Participants: Hundred and ninety-eight people with MCI or early-stage dementia were included.

Intervention: The intervention group $(n=100)$ received 11 individual weekly sessions of the CORDIAL program. This intervention includes elements from CBT, cognitive rehabilitation, and reminiscence therapy. The control group $(n=98)$ received treatment-as-usual.

Measurements: We assessed Montgomery-Åsberg Depression Rating Scale (MADRS) (main outcome), Neuropsychiatric Inventory Questionnaire, and Quality of Life in Alzheimer's disease (secondary outcomes) over the course of 4 months and at a 10-month follow-up visit.

Results: A linear mixed model demonstrated that the depressive symptoms assessed by MADRS were significantly more reduced in the intervention groups as compared to the control group $(p<0.001)$. The effect persisted for 6 months after the intervention. No significant differences between groups were found in neuropsychiatric symptoms or quality of life.

Conclusion: Our multicomponent intervention, which comprised 11 individual sessions of CBT, cognitive rehabilitation, and reminiscence therapy, reduced depressive symptoms in people with MCI and dementia.

Key words: dementia, mild cognitive impairment, cognitive rehabilitation, cognitive behavioral therapy, reminiscence therapy, psychosocial intervention, depressive symptoms, MADRS

Clinical Trial Registration: NCT02013518

Correspondence should be addressed to: Johanne Bjoernstad Tonga, Oslo University Hospital, Department of Old Age Psychiatry, Postbox 4956, Nydalen, 0424 Oslo, Norway, Phone: +0047 23163000; Fax: +004723163002. Email: jotbjo@ ous-hf.no. Received 14 May 2019; revision requested 04 Sep 2018; revised version received 24 Jan 2020; accepted 27 Jan 2020. First published online 05 March 2020.

\section{Introduction}

After a diagnosis of dementia, patients often report losing friends and feeling lonely, anxious, distressed, and depressed (Alzheimer's Association, 2014; 
Harman and Clare, 2006). Depression occurs in up to two-thirds of all patients (Enache et al., 2011). Research suggests that people with mild cognitive impairment (MCI) and dementia exhibit an increased risk for developing depression compared to people with normal cognitive function; at 4-year follow-up, 38\% people with MCI and 43\% with dementia reported depression, compared to $20 \%$ people with normal cognition (Snowden et al., 2015). Furthermore, research has shown that depressive symptoms substantially impacts the disease course, because it leads to reduced independence, increased risk of institutionalization, and a higher caregiver burden (Orgeta et al., 2015). Therefore, psychosocial interventions designed to reduce depressive symptoms should support the patient in understanding the diagnosis and making positive adjustments (Lee et al., 2014).

Previous research on psychosocial treatments in people with MCI or dementia found that psychological interventions could effectively reduce depression (Orgeta et al., 2015). Consistent with that finding, a meta-analysis of psychosocial interventions also found significant reductions of depressive symptoms with a medium effect size in residents of long-term care (Cody and Drysdale, 2013). Specifically, cognitive behavioral therapy (CBT), which focuses on behavioral activity scheduling, significantly reduced depressive symptoms in people with dementia that lived at home (Teri et al., 1997; Teri et al., 2005) or in a nursing home (Hyer et al., 2009). Reminiscence therapy that involves reflecting on past activities, events, and experiences, typically elicited with prompts, like photographs or music, effectively improved cognitive function and mood in people with dementia (Cotelli et al., 2012). These findings were supported by a meta-analysis of randomized controlled trials, which demonstrated that reminiscence therapy had a small effect size on cognitive functions and a moderate effect size on depressive symptoms (Huang et al., 2015). In contrast, two other studies failed to demonstrate positive findings with reminiscence therapy on cognition and mood (Wang, 2007; Woods et al., 2012). In addition, cognitive rehabilitation, which focuses on intact memory skills and learning compensatory strategies for managing memory problems, had a positive effect on mood and memory performance in people with MCI (Kurz et al., 2009). Another cognitive rehabilitation intervention study found significant effects on goal attainment for people with dementia, but no differences between the intervention and control group were observed in mood, cognition, quality of life, or self-efficacy (Clare et al., 2019). In addition, a large multicenter randomized controlled trial (ETNA3 study) compared three different interventions to treatment-as-usual for patients with Alzheimer's disease; (1) group therapy of cognitive training, (2) group therapy of reminiscence, and (3) individualized cognitive rehabilitation. They found that only the individualized cognitive rehabilitation provided clinically significant results and recommend that interventions should be individualized to meet each patient's needs.

Studies have also suggested that transferring intervention results to everyday life could be facilitated by involving caregivers, due to more frequent practicing between therapy sessions (Brodaty et al., 2003; Clare et al., 2010). Furthermore, because people with dementia constitute a heterogeneous group, broad interventions with multicomponent treatments are needed. A systematic review by Olazarán et al. (2010) found that multicomponent interventions were effective in improving mood and quality of life among people with dementia. They also found that multicomponent interventions that provided caregiver education and support delayed the institutionalization of people with dementia. Other multicomponent treatments, which included cognitive rehabilitation and cognitive behavioral group therapy for people with MCI, have found increased acceptance of their cognitive condition in the intervention group compared to the waiting-list control (Banningh et al., 2011).

The cognitive rehabilitation and cognitive behavioral treatment for early dementia (CORDIAL) program comprised CBT, reminiscence therapy, and cognitive rehabilitation. In a randomized controlled trial, called the CORDIAL study, this multicomponent intervention significantly reduced depressive symptoms among female participants, based on the Geriatric Depression Scale (GDS). However, they found no significant effect on activities of daily life (ADL) functions (Kurz et al., 2012). Later, the same group (Werheid et al., 2015) reanalyzed the effects of the CORDIAL program on depressive symptoms with latent change score modeling. They demonstrated that the CORDIAL program reduced depressive symptoms by 1.5 points, on the GDS, and the effect remained stable, but it was restricted to females. Moreover, the program had a pronounced antidepressive effect in patients with clinically relevant depressive symptoms at baseline. Another study used the CORDIAL manual in a group setting and found a positive interaction effect on quality of life (Brueggen et al., 2017). They found no effect on ADL functions and they did not assess depressive symptoms. Those authors suggested that ADL functions might be difficult to assess as a primary outcome, because dementia is a progressive disease.

To summarize, although psychosocial interventions have shown promise for people with dementia, there remains a need for more multicomponent, 
well-designed trials with adequate power and appropriate outcome measures (Olazarán et al., 2010).

\section{Purpose of the study}

In the present randomized controlled trial, we examined the effects of a multicomponent treatment (the CORDIAL program) on depressive symptoms in people with MCIs and mild dementia, since previous research on the CORDIAL program had shown promising effects on depressive symptoms (Werheid et al., 2015). We hypothesized that the CORDIAL program would significantly reduce depressive symptoms, assessed by MontgomeryÅsberg Depression Rating Scale (MADRS), compared to treatment-as-usual (control).

\section{Methods}

\section{Trial design}

This multicenter randomized controlled trial randomly allocated participants to receive either treatment-as-usual $(n=98)$ or the CORDIAL intervention $(n=100)$.

\section{Participants}

Eligible participants had to:

- meet the Winblad criteria for MCI due to Alzheimer's disease (Winblad et al., 2004); or criteria for dementia in Alzheimer's disease according to the ICD-10 or the National Institute of Neurological and Communicative Disorders and Stroke and the Alzheimer's Disease and Related Disorders Association (Dubois et al., 2007);

- be in the early stages of MCI or dementia, as indicated by a Mini Mental State Examination (MMSE) (Folstein et al., 1975) score of 20 or above;

- reside at home;

- be in weekly contact with a caregiver that was willing to participate, for example, a spouse, friend, sibling, or adult child;

- informed consent from the participants and caregivers.

Individuals were excluded, when they:

- lived outside the home, for example, a nursing home;

- received ongoing psychotherapy;

- had a severe psychiatric or somatic illness that could hinder study adherence.

- Participants that were taking ongoing medication in stable doses were not excluded, due to ethical and practical considerations.

Settings and data collection methods Participants were recruited from five old age psychiatry and memory clinics in Norway, from January 2013 to June 2017. All participants underwent a comprehensive assessment, including advanced cognitive testing, which followed a standard protocol (Brækhus et al., 2011); brain MRI scans, for measuring the hippocampus; and spinal fluid tests, for determining tau and amyloid- $\beta$ protein levels. On MRI scans, the hippocampus was measured either by visual inspection (Scheltens et al., 1992) or with volumetric calculations performed with Neuroquant software (Brewer et al., 2009; Engedal et al., 2012). The final MCI or dementia diagnosis included the MRI findings and the tau and amyloid$ß$ protein results. Depression diagnosis was evaluated according to the ICD-10 criteria. Diagnoses were determined by reaching a consensus among experienced geriatricians, a psychiatrist, a neurologist, and in some cases, a neuropsychologist. Based on this comprehensive assessment, people with MCI or dementia that met the inclusion criteria were asked to participate, either verbally, in participating clinics, or through a letter, followed by a phone call.

\section{Baseline assessments}

All participants completed standardized baseline questionnaires. This covered background characteristics and structured measures of cognitive and psychiatric symptoms (which are detailed described under the subsection "Outcomes"). Next, they were allocated to the intervention or control group. Participants also completed assessments at the end of the RCT; 4 months after baseline and at the 6-month follow-up (10 months after baseline) (if they did not drop out). All standardized assessments were administered by educated health staff, nurses, psychologists, psychiatrist, occupational therapists, and master students in psychology. They were trained in performing cognitive testing and interviews, according to a written manual, and they received regular supervision.

If the patient fulfilled the criteria for depression according to ICD-10, the depression diagnosis was made. Also, participants were categorized into severity groups, based on the MADRS, where: $0-8=$ no depression; 9-17 = mild depression; $18-34=$ moderate depression; and $\geq 35=$ severe depression (Mittmann et al., 1997; Müller et al., 2000).

\section{Interventions}

We based the intervention on that described in the German CORDIAL study (Kurz et al., 2012), but we adapted it to Norwegian culture, after conducting a pilot study (Tonga et al., 2015; 2016). The main modifications were: (1) participants completed 11 instead of 12 sessions; (2) depressive symptoms were treated before memory aids were given, because we assumed it would be easier to motivate patients to try memory aids and reminiscence work if 
Table 1. Overview of treatment-as-usual $(N=85)$

\begin{tabular}{|c|c|c|}
\hline TREATMENT & $N$ & $\begin{array}{c}\text { PERCENTAGE } \\
(\%)\end{array}$ \\
\hline $\begin{array}{l}\text { Routine follow-ups, including assessments performed by a doctor or nurse at the existing } \\
\text { memory clinics and services offered by the community, such as day center support } \\
\text { or activity groups }\end{array}$ & 14 & 16.7 \\
\hline Group therapy: eight sessions of CBT and ACT & 8 & 9.5 \\
\hline Other follow-ups, not specified & 14 & 16.7 \\
\hline No treatment/no follow-up & 48 & 57.1 \\
\hline
\end{tabular}

Note. Treatment-as-usual was only reported by the 85 participants that completed the 4-month post-treatment assessment. Thus, 13 participants were lost to follow-up

Abbreviations: CBT, cognitive behavioral therapy; ACT, acceptance and commitment therapy.

they initially had learned some techniques about how to handle depressive symptoms; (3) electronic memory aids were used; (4) the pleasant activity schedule and life review assignments were modified to include more typically male activities, such as soccer, fishing; and (5) we shortened the caregiver psychoeducational information.

Inspired by cognitive therapy, the intervention included a clear agenda in every session, Socratic questioning, specific worksheets, homework inbetween sessions, and psychoeducation about dementia (Beck, 2011). Module 1 explained the intervention and explored the patient's problem and treatment goals, consistent with classical cognitive therapy (Beck, 2011). Module 2 focused on structuring the day and establishing regular pleasant activities to reduce depressive symptoms, similar to the approach of Teri et al. (1997). Modules 3 and 4 were inspired by Clare et al. (2010); they provided cognitive rehabilitation by building on existing memory skills, learning compensatory memory aids, and using behavioral routines to reduce the demand on memory. Module 5 was inspired by Woods et al. (2018); it provided reminiscence therapy with biographical materials selected to increase the patient's mood and well-being. The closing module, module 6 , included a review of the program, an evaluation of individual goals, and suggestions for how to maintain the positive outcomes of therapy. An overview of the intervention is given in Figure 2.

Patients with MCI or dementia participated in all 11 sessions. All caregivers participated in every other session (six sessions). This approach aimed to aid the patient in transferring the newly learned strategies into everyday life and it provided information on MCI and dementia to the caregiver. Additionally, caregivers received letters that described the background and content of each module.

The therapists comprised nurses, psychiatrists, occupational therapists, and psychologists with training and experience in CBT. Therapists were instructed to adhere to the manual, but they were given flexibility in distributing the time between treatment modules and in tailoring the therapy to each patient's needs. Also, all therapist received training in the CORDIAL intervention before the intervention started and did participate in regular supervision and workshops. The therapists selfrated treatment adherence after every session based on structured questions.

In the control group, treatment-as-usual was not standardized. Of the 98 people in the control group, $57 \%$ reported no follow-up assessments and the remaining $43 \%$ received various types of followup assessments (Table 1).

\section{Outcomes}

BACKGROUND VARIABLES

We recorded the age, gender, kinship, work situation, years of schooling, and hobbies for people with dementia and caregivers. In addition, we recorded the duration of symptoms, current medications, and formal treatments, measured with the Resource Utilization in Dementia Questionnaire - Lite version (Wimo and Winblad, 2003). We recorded changes in medication and major life events at the 4- and 10-month assessments.

Cognitive function was assessed by trained health staff, with the Norwegian version of the Mini Mental State Examination (MMSE-NR) (Strobel and Engedal, 2008). This instrument is commonly used as a screening tool for dementia and it covers different cognitive domains. Previously, Folstein et al. (1975) examined the reliability and validity of the MMSE and they reported a Cronbach's alpha of 0.89. In addition, the ability to perform ADL was proxyrated by their caregiver with the Instrumental Activities of Daily Living Scale (I-ADL) (Lawton and Brody, 1969), which measures everyday 
independent activities, such as cooking, cleaning, and managing finances. The score ranges from 1 (independent) to 5 (totally dependent), with a maximum total score of 31 . Previous studies reported Cronbach's alphas for the I-ADL that ranged from 0.78 to 0.91 (Sikkes et al. 2009).

\section{Patient variables}

Depressive symptoms were measured with the MADRS (Montgomery and Åsberg, 1979), administered by professional health staff, and was the primary outcome of the study. The MADRS comprises 10 items that cover the most common depressive symptoms reported by patients, including sadness, inner tension, reduced sleep, and suicidal thoughts. Responses were scored based on the clinical judgment of the severity by the interviewer on a scale ranging from 0 to 6 , with a maximum total score of 60. Fantino and Moore (2009) reported a Cronbach's alpha of 0.84 for the MADRS. The questionnaire has been validated in people with dementia and is considered a consistent tool for detecting depression in dementia independently of the severity of the dementia disease (MüllerThomsen et al. 2005).

Neuropsychiatric symptoms were proxy-rated by caregivers with the short form of the Neuropsychiatric Inventory Questionnaire (NPI-Q) (Kaufer et al., 2000). The questionnaire consisted of 12 items that covered several symptoms, including apathy, aggression, anxiety, depression, hallucinations, and sleeping difficulty. It also included a severity sum score that ranged from 0 to 36, where a higher score indicated more severe symptoms. The NPI-Q has been translated into Norwegian (Årsland \& Dramsdahl) and it was reliability-tested and validated in patients living in a nursing home (Selbaek et al., 2008).

At the 4-month assessment, people with dementia self-reported responses to the question: "Overall, how are you coping with everyday life after participating in the study?". The responses ranged from 1 to 4 , where $1=$ no difference and $4=$ improved coping.

\section{Caregiver VARIABles}

Anxiety and depression were self-reported by the caregivers with the Hospital Anxiety and Depression Scale (HADS) (Zigmond and Snaith, 1983). Each item was rated on a four-point scale that ranged from 0 to 3 and the maximum total score was 42 . A cutoff score $>8$ indicated substantial anxiety and depressive symptoms (Bjelland et al., 2002). A meta-study by Bjelland et al. (2002) reported that Cronbach's alphas ranged from 0.68 to 0.93 for the HADS.
The burden of caregivers was self-reported with the Relative Stress Scale (RSS) (Greene et al., 1982). This questionnaire consisted of 15 questions, rated from 0 (not at all) to 4 (to a high degree), and the total score ranged from 0 to 60 . The Cronbach's alpha ranged between 0.70 to 0.86 (Ulstein et al., 2007).

Patient And Caregiver Questionnaires Quality of life was recorded in a structured interview conducted by trained professionals with the Norwegian version of the Quality of life in Alzheimer's disease (QOL-AD) instrument (Logsdon et al., 1999). This 13-item questionnaire covered quality of life in different domains, including living conditions, physical health, relationships, and financial conditions. Each item was rated on a four-point scale, and the total score ranged from 13 to 52 . The QOL-AD was systematically examined for people with dementia and it showed satisfactory validity. The estimated Cronbach's alpha was 0.82 (Thorgrimson et al., 2003).

Self-efficacy was self-reported with the General Self-Efficacy (GSE) scale, which included 10 items (Schwarzer et al., 1997). Participants responded on a four-point scale, as follows: not at all true, hardly true, moderately true, and exactly true. The scale ranged from 10 to 40 , with higher values indicating higher self-efficacy. In a sample that covered 23 nations, the estimated Cronbach's alphas ranged from 0.76 to 0.90 (Schwarzer et al., 1997).

\section{Sample size}

The sample size was calculated for independent samples t-tests, a significance level of 0.05 and $80 \%$ power. Based on a study performed at the memory clinic in Oslo including 520 early-stage dementia patients with a mean MADRS score of 9.7 (standard deviation [SD] 6.7) (Knapskog et al., 2011), 79 persons were needed in each group $(\mathrm{N}=158)$ with an expected decrease of 3 points in the intervention group and no change in the control group. However, a high dropout rate is realistic in studies with elderly people because of illness, death, or cognitive impairment (Chatfield et al., 2005). Therefore, the study allowed for a $20 \%$ dropout rate, indicating 98 patients in each group. Thus, the study had sufficient power, which decreases the possibility of a type II error (Field, 2013).

RANDOMIZATION

Identity numbers that assigned to participants were entered into a computer-generated randomization program (http://randomization.com/). Then, we performed block randomization, with four participants 
in each group (intervention or control). The randomization was kept blinded for the research team.

\section{Statistical methods}

Baseline characteristics of patients in each group and for the total sample are presented as mean and SD for continuous variables, and as frequency and percentage for categorical variables. To evaluate differences in characteristics between patients that completed and those that discontinued the study, we performed an independent samples t-test, for continuous variables, and a chi square test, for categorical variables.

Because patients were recruited from different centers, the data might exhibit a hierarchical structure. Moreover, due to repeated measurements for each patient, intra-patient correlations were likely to be present. Therefore, we assessed differences between the intervention and control groups in time trend in primary (MADRS) and secondary (NPI-Q and QOL-AD) outcome variables with a linear mixed model, one for each variable. The models contained fixed effects for nonlinear time, the treatment group, and interactions between the two. A significant interaction would imply overall trend differences between the groups. Random effects were included for patients nested within centers. Models were adjusted for patient GSE, caregiver GSE, and caregiver RSS scores. Also, age and gender were included as confounders. We applied Akaike's information criterion (AIC) to reduce the multiple models regarding adjustment variables and performed standard residual diagnostics and found no serious violations of model assumptions. Differences between the groups at each time point were derived in post hoc analyses. In addition, longitudinal analysis of covariance (ANCOVA) was performed, as a sensitivity analysis, to test whether baseline differences impacted the results. We calculated Cohen's $d$ for between-group differences at each time point with the corresponding $95 \%$ confidence intervals (CIs). Data are presented as regression coefficients and standard errors and illustrated graphically as trend curves with $95 \%$ CIs at each time point. Residual diagnostic did not show any larger deviations from model assumptions. All analyses were performed with SPSS v25 and SAS v9.4. $p$-Values $<0.05$ were considered statistically significant.

\section{Ethics}

Written informed consent was obtained from all patients and caregivers. The study followed the ethical principles outlined in the Declaration of Helsinki and it was approved by the Regional Committee for Ethics in Medical Research, South-Eastern Norway.

\section{Results}

\section{Participant selection}

The CONSORT diagram in Figure 1 details the patient selection and allocation procedures. The dropout rate from baseline to 4 months postintervention was $14 \%$ (27 of 198) and the dropout rate from 4 to 10 months post-intervention was $20 \%$ (40 of 198). Attrition analyses of participants that completed compared to participants that discontinued the study showed no significant differences in background variables between groups $(p>.05)$.

\section{Baseline data}

The ratio of dementia to MCI was higher in the intervention group than in the control group (Table 2). We found significant baseline differences between participants with MCI and dementia, on the I-ADL (mean scores, MCI: 11.5; dementia: 13.3; $p=0.009$ ) and the MMSE (mean scores, MCI: 25.3; dementia: 24.2; $p=0.012$ ). However, these groups were not significantly different on the MADRS, GSE, QOL-AD, or NPI, and they had similar hobbies, ages, genders, marital statuses, schooling, formal help, work situations, and symptom durations.

Overall, female participants were underrepresented in both the control and intervention groups. Most participants received no formal assistance, were relatively independent, and had a high level of cognitive function. High mean scores on the QOL-AD and GSE and low mean scores on the MADRS suggested that participants functioned well, with few patients score in the range of severe depressive symptoms. Most participants used medication on a daily basis $(90.9 \%) ; 42.9 \%$ reported anti-dementia medications and $14.1 \%$ reported antidepressant medications. No changes in medication were reported at the 4-month post-intervention assessment, which indicated that medication doses remained stable during the trial.

Most caregivers were female and had a hobby. High mean scores on the QOL-AD and GSE and low mean scores on the HADS and RSS suggested that caregivers had few symptoms and a high quality of life.

\section{Outcomes}

According to unadjusted linear mixed model, the intervention group exhibited significantly larger reduction in the primary outcome, MADRS, than control group $(p<0.001$ for interactions; Figure 3). According to post hoc analyses, the groups were different at baseline (T0) $(p=0.017)$, but not at 4 months post-intervention (T4) or at the follow-up assessment (T10). Cohen's $d$ values (95\% CIs) at T0, T4, and T10 were -0.4 


\section{CONSORT 2010 Flow Diagram}

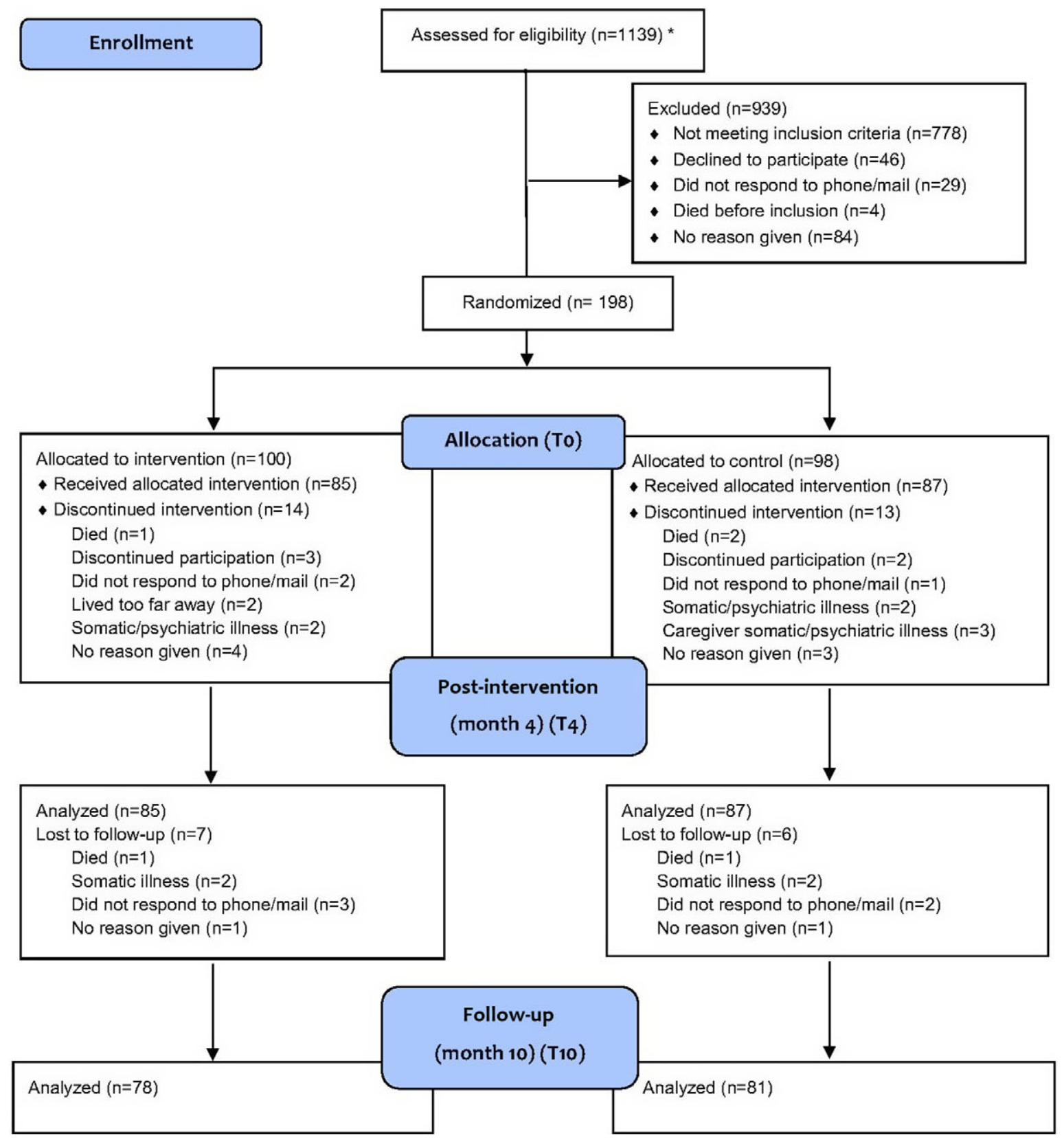

Fig. 1. Flowchart of the selection, randomization, and analyses of people with $\mathrm{MCl}$ and dementia and their caregivers. *Only two of the four participating hospitals systematically reported eligibility assessments. Thus, the numbers reflect only the procedures and assessments conducted in two centers. However, this accounted for $85 \%$ of the participants.

$(-0.7 ;-0.1), 0.1(-0.2 ; 0.5)$, and $-0.1(-0.5 ; 0.2)$, respectively.

In a multiple AIC-reduced model, the overall difference in change between groups was still significant ( $p=0.001$ for interactions; Table 3), but post hoc analyses did not show any difference between groups at different time points. According to the longitudinal ANCOVA adjusted for covariates, the results were the same as by the linear mixed model.

Secondary outcomes were also analyzed with both the linear mixed model and a longitudinal ANCOVA adjusting for baseline differences. Both models showed no significant differences in overall trend between the control and intervention group in quality of life for people with dementia or their 


\begin{tabular}{|ll|}
\hline Module 1 & Introduction \\
\hline 1st session* & Introduction and treatment goals \\
\hline Module 2 & Pleasant activities \\
\hline 2nd session & Identify pleasant activities and schedule pleasant events \\
3rd session* & Engage in a pleasant activity with the caregiver \\
\hline Module 3 & Memory aids \\
\hline 4th session & Introduction to memory aids \\
5th session* & Use memory aids with the caregiver \\
\hline Module 4 & Daily routines \\
\hline 6th session & Establish daily memory routines \\
7th session* & Complete daily memory routines with the caregiver \\
\hline Module 5 & Reminiscence \\
\hline 8th session & Introduce biographical material \\
9th session* & Review biographical material with the caregiver \\
\hline Module 6 & Closing module \\
\hline 10th session & Review the program and discuss ways to continue with the skills learned \\
\hline Closing session with the caregiver \\
\hline (th
\end{tabular}

Fig. 2. An overview of the intervention modules. Homework assignments were completed in-between sessions. *Both the caregiver and the patient participated in the session.

caregivers, the neuropsychiatric symptoms in people with dementia, or depressive symptoms and anxiety symptoms in caregivers.

Furthermore, in AIC-reduced multiple models, a higher patient GSE score was associated with less depressive symptoms (MADRS; $p<0.001$ ), better patient quality of life $(p<0.001)$, but lower caregiver quality of life $(p=0.038)$. A higher caregiver GSE score was significantly associated with less caregiver depressive symptoms (MADRS; $p=0.033$ ), lower caregiver HADS scores $(p<0.001)$, and better caregiver quality of life $(p<0.001)$. A higher caregiver RSS score was significantly associated with higher HADS scores $(p<0.001)$, a worse caregiver quality of life $(p<0.001)$, and more neuropsychiatric symptoms in people with dementia $(p<0.001)$. Also, female patients had more depressive symptoms (MADRS; $p=0.005)$ and higher NPI scores $(p=0.004)$, compared to male patients.

At 4 months after the baseline assessment, people with dementia in the intervention group scored significantly higher than the control group on the question "overall, how are you coping with everyday life, after participating in the study?" ( $p<0.001$, chi square test).

\section{Discussion}

The main finding of this study was that the CORDIAL intervention affected depressive symptoms compared to treatment-as-usual and this effect was maintained for 6 months after the intervention. This positive finding was consistent with previous findings with other psychosocial interventions that significantly reduced depressive symptoms in people with dementia (Cotelli et al., 2012; Hyer et al., 2009; Kurz et al., 2009; Kurz et al., 2012; Teri et al., 1997; Woods et al., 2018). For example, Teri et al. (1997) demonstrated that behavioral treatment for patients with dementia living at home significantly reduced depression and the effect was maintained at the 6month follow-up. However, in contrast to Teri et al. (1997), most patients in the present study did not qualify for clinical depression. Rather, they exhibited depressive symptoms, which indicated that this multicomponent treatment was effective for the more subtle depressive symptoms observed in people with MCI and early dementia. Additionally, a previous German CORDIAL intervention performed by Kurz et al. (2012) reported a significant change in depression, but the effect was restricted to 
Table 2. Baseline characteristics of 198 people with dementia and their caregivers; $n=80$ people with amnestic mild cognitive impairment $(\mathrm{MCl})$ due to Alzheimer's disease $(40 \%) ; n=118$ people with dementia (60\%)

\begin{tabular}{|c|c|c|c|}
\hline CHARACTERISTIC & $\begin{array}{c}\text { INTERVENTION } \\
\quad(N=100)\end{array}$ & $\begin{array}{l}\text { CONTROL } \\
(N=98)\end{array}$ & $\begin{array}{c}\text { THE WHOLE SAMPLE } \\
(N=198)\end{array}$ \\
\hline \multicolumn{4}{|l|}{$\begin{array}{l}\text { People with dementia or } \\
\text { amnestic MCI }\end{array}$} \\
\hline Age, years, mean (SD) & $69.4(7.79)$ & $70.7(7.84)$ & $70.1(7.82)$ \\
\hline \multicolumn{4}{|l|}{ Gender, $n(\%)$} \\
\hline Females & $45(45 \%)$ & $47(48 \%)$ & $92(47 \%)$ \\
\hline Males & $55(55 \%)$ & $51(52 \%)$ & $106(53 \%)$ \\
\hline Duration of symptoms & [97] & [96] & [193] \\
\hline $\begin{array}{l}\text { (years), }[n]^{\mathrm{a}} \text {, } \\
\text { mean }(\mathrm{SD})\end{array}$ & $3.1(1.74)$ & $3.1(2.47)$ & $3.1(2.2)$ \\
\hline \multicolumn{4}{|l|}{ Diagnosis, $n(\%)$} \\
\hline Mild cognitive & $32(32 \%)$ & $48(50 \%)$ & $80(40 \%)$ \\
\hline impairment & $68(68 \%)$ & $48(50 \%)$ & $118(60 \%)$ \\
\hline \multicolumn{4}{|l|}{ Dementia diagnosis } \\
\hline \multicolumn{4}{|l|}{ Depression severity, $n(\%)$} \\
\hline Not depressed & $58(58 \%)$ & $71(72.4 \%)$ & $129(65.2 \%)$ \\
\hline Mild & $33(33 \%)$ & $21(21.4 \%)$ & $54(27.3 \%)$ \\
\hline Moderate & $8(8 \%)$ & $5(5.1 \%)$ & $13(6.6 \%)$ \\
\hline Severe & $1(1 \%)$ & $1(1 \%)$ & $2(1 \%)$ \\
\hline Received formal help, $[n]$, & [90] & [91] & [181] \\
\hline Yes, $n(\%)$ & $14(16 \%)$ & $11(12 \%)$ & $25(28 \%)$ \\
\hline No, $n(\%)$ & $76(84 \%)$ & $80(88 \%)$ & $156(172 \%)$ \\
\hline \multicolumn{4}{|l|}{ Marital status, $n(\%)$} \\
\hline Married & $83(83 \%)$ & $81(82 \%)$ & $162(83 \%)$ \\
\hline Widowed & $6(6 \%)$ & $5(5 \%)$ & $11(8 \%)$ \\
\hline Single & $11(11 \%)$ & $12(12 \%)$ & $23(12 \%)$ \\
\hline Schooling (years), $[n]$, & [99] & [95] & [194] \\
\hline mean $(\mathrm{SD})$ & $13.9(3.17)$ & $13.5(3.17)$ & $13.8(3.3)$ \\
\hline \multicolumn{4}{|l|}{ Work situation, $n(\%)$} \\
\hline Currently working & $9(9 \%)$ & $9(9 \%)$ & $18(9 \%)$ \\
\hline Sick leave & $7(7 \%)$ & $8(8 \%)$ & $15(8 \%)$ \\
\hline Retired & $84(84 \%)$ & $81(82 \%)$ & $162(83 \%)$ \\
\hline Hobbies, $[n]$, & [97] & [98] & [194] \\
\hline Yes, $n(\%)$ & $62(64 \%)$ & $70(72 \%)$ & $132(67 \%)$ \\
\hline No, $n(\%)$ & $35(36 \%)$ & $28(29 \%)$ & $62(32 \%)$ \\
\hline \multicolumn{4}{|l|}{ MMSE, } \\
\hline mean $(\mathrm{SD})$ & $24.7(3.16)$ & $24.5(2.95)$ & $24.6(3.1)$ \\
\hline \multicolumn{4}{|l|}{ GSE, } \\
\hline mean $(\mathrm{SD})$ & $26.9(6.37)$ & $29.7(6.88)$ & $28.3(6.7)$ \\
\hline I-ADL, $[n]$, & [97] & [98] & [195] \\
\hline mean $(\mathrm{SD})$ & $12.7(4.72)$ & $12.6(4.88)$ & $12.7(4.80)$ \\
\hline NPI, $n$, & [98] & [98] & [196] \\
\hline mean (SD) (IQR) & $3.2(2.55)$ & $2.8(2.36)$ & $3(2.46)$ \\
\hline \multicolumn{4}{|l|}{ QOL-AD, } \\
\hline mean (SD) & $37.7(5.46)$ & $39.7(5.31)$ & $7.6(6.40)$ \\
\hline \multicolumn{4}{|l|}{ MADRS, } \\
\hline mean $(\mathrm{SD})$ & $8.8(6.32)$ & $6.5(6.23)$ & $7.6(6.40)$ \\
\hline \multicolumn{4}{|l|}{ Caregivers } \\
\hline \multicolumn{4}{|l|}{ Age, } \\
\hline mean (SD) & $66.8(11.10)$ & $65.7(11.98)$ & $66.2(11.54)$ \\
\hline \multicolumn{4}{|l|}{ Gender, $n(\%)$} \\
\hline Females & $66(66 \%)$ & $67(68 \%)$ & $133(134 \%)$ \\
\hline Males & $34(34 \%)$ & $31(32 \%)$ & $65(66 \%)$ \\
\hline Hobbies, $[n]$, & [95] & [96] & [191] \\
\hline Yes, $n(\%)$ & $71(74 \%)$ & $68(70 \%)$ & $139(72.3 \%)$ \\
\hline No, $n(\%)$ & $24(25 \%)$ & $28(29 \%)$ & $52(27.2)$ \\
\hline
\end{tabular}


Table 2. Continued

\begin{tabular}{|c|c|c|c|}
\hline CHARACTERISTIC & $\begin{array}{l}\text { INTERVENTION } \\
\quad(N=100)\end{array}$ & $\begin{array}{c}\text { CONTROL } \\
(N=98)\end{array}$ & $\begin{array}{c}\text { THE WHOLE SAMPLE } \\
(N=198)\end{array}$ \\
\hline HADS, [n], & [97] & [98] & [193] \\
\hline mean (SD) & $8.5(5.9)$ & $5.8(5.2)$ & $7.1(5.6)$ \\
\hline QOL-AD, $[n]$, & [98] & [97] & [195] \\
\hline Mean (SD) & $40.5(5.43)$ & $42.0(5.90)$ & $41.2(5.66)$ \\
\hline GSE, $[n]$, & [98] & [97] & [195] \\
\hline Mean (SD) & $30.4(6.08)$ & $31.7(6.25)$ & $62.1(6.17)$ \\
\hline $\mathrm{RSS},[n]$ & [98] & [97] & [195] \\
\hline mean (SD) & $14.3(11.1)$ & $11.1(9.7)$ & $12.7(10.4)$ \\
\hline
\end{tabular}

a $n]$ indicates the numbers of participants, when data were missing.

Abbreviations: SD, standard deviation; MMSE, Mini Mental State Examination; GSE, General Self-Efficacy Scale; I-ADL, Instrumental Activities of Daily Living; NPI, Neuropsychiatric Inventory; MADRS, Montgomery-Asberg Depression Rating Scale; HADS, Hospital Anxiety and Depression Scale; QOL-AD, Quality of Life in Alzheimer's disease; RSS, Relative Stress Scale.

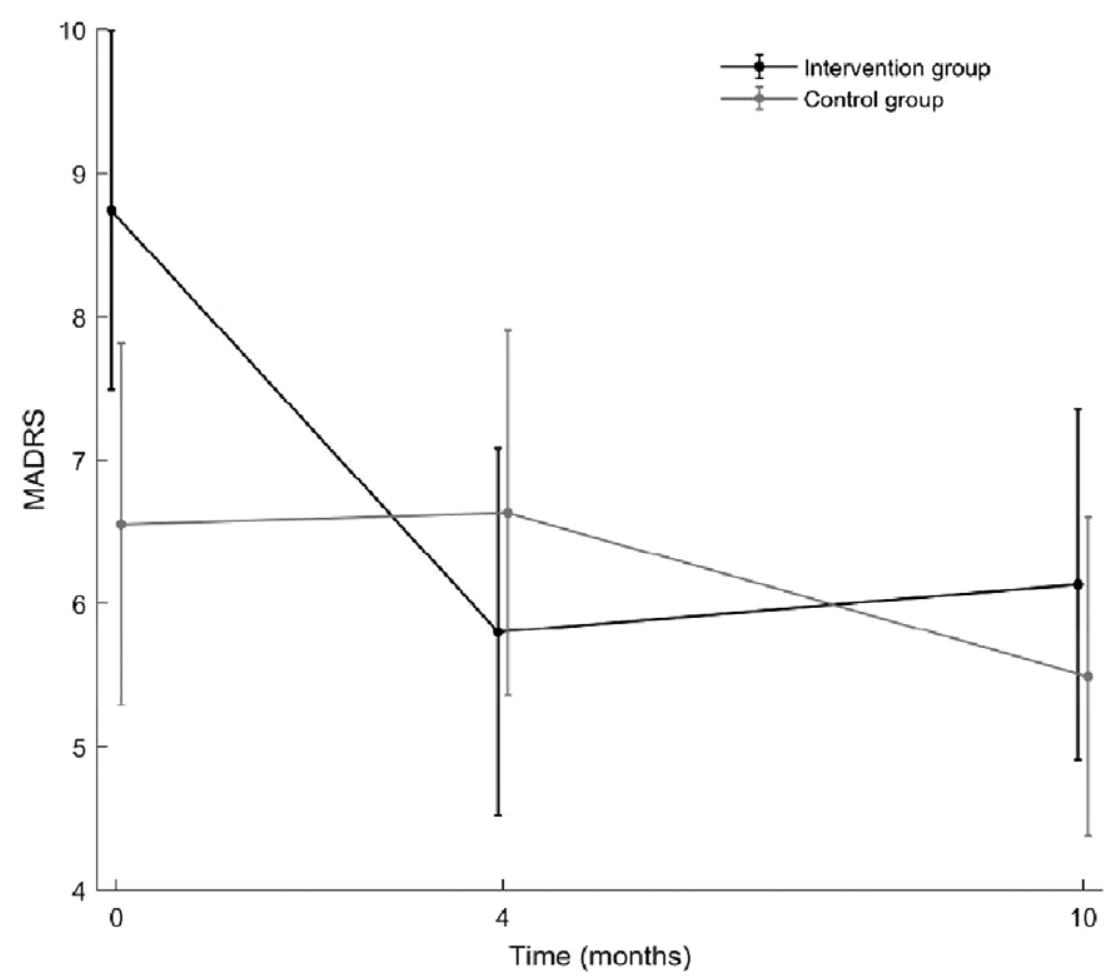

Fig. 3. Illustration of the trends in the measurement of depressive symptoms (MADRS) over time. Black line: intervention group, gray line: control group. Results are from an unadjusted linear mixed model. MADRS = Montgomery-Åsberg Depression Rating Scale.

female participants. In contrast, the present study found significant group changes in depressive symptoms for both female and male participants. This discrepancy between studies might be explained by the use of different depressive outcome measures; the study by Kurz et al. (2012) used the GDS, which only included yes/no questions; in contrast, the present study employed the MADRS, which consists of 10 items each rated from 0 to 6 based on the clinician judgment of the severity. Another explanation might be that the present study modified the intervention somewhat, by including more typical male activities in the pleasant event schedule, such as soccer, fishing, driving a car. However, in contrast to the present study, other studies did not find significant changes in depressive symptoms among people with MCI and dementia (Banningh et al., 2011; Clare et al., 2019; Stockwell-Smith et al., 2018; Wang, 2007; Woods et al., 2012). Potential explanations might be differences in the psychosocial interventions employed, the outcome measurements, and/or the baseline characteristics of participants. 


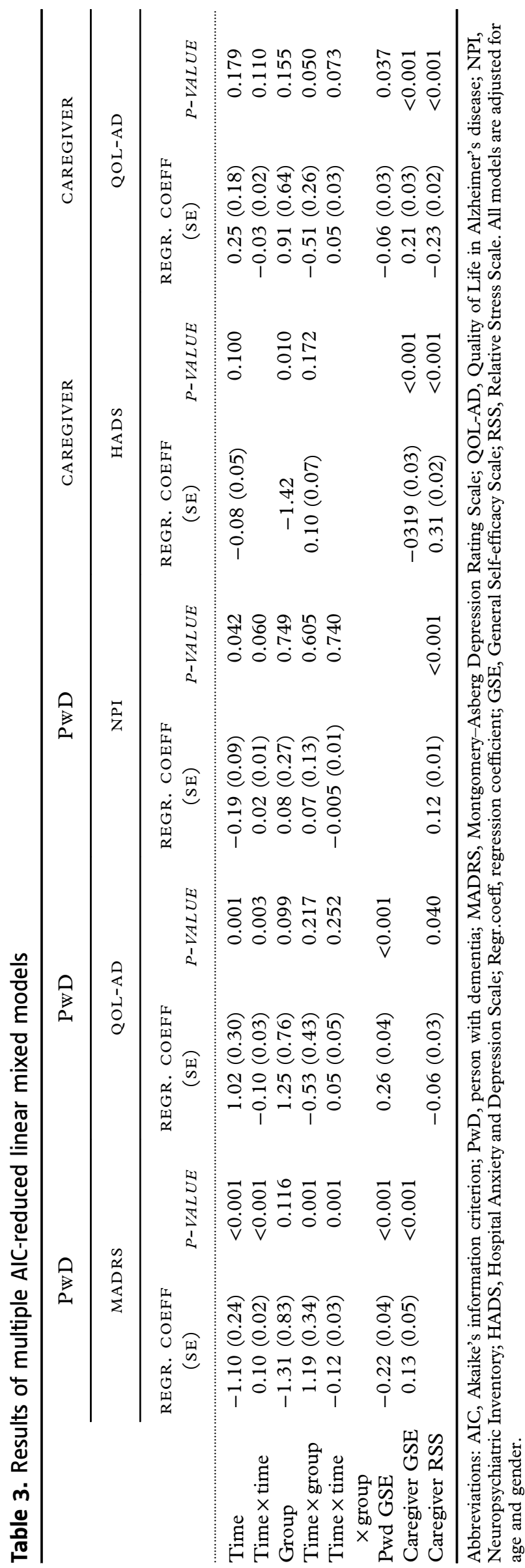

Another promising finding of the present study was that we observed significant changes in coping with everyday life in the intervention group at the 4-month assessment, after the intervention. This finding was consistent with findings from another study that tested a multicomponent group intervention for people with MCIs, which reported improved coping in the intervention group (Banningh et al., 2011). However, more advanced statistics and outcome measures of coping are necessary to confirm that finding.

In contrast to studies that used other psychosocial interventions and reported positive quality of life effects (Brueggen et al., 2017; Olazarán et al., 2010), the present study did not find any significant group changes in either of the two secondary outcomes, neuropsychiatric symptoms, or quality of life. This was surprising, because one would expect that reducing depressive symptoms would lead to positive interactions with other variables. Other studies have demonstrated a strong relationship between depression and quality of life (Kim and Shim, 2015) and a strong relationship between neuropsychiatric symptoms and depression (Prado-Jean et al., 2010). However, consistent with our findings, other psychosocial intervention studies failed to find significant changes in the quality of life or neuropsychiatric symptoms (Appelhof et al., 2019; Clare et al., 2019; Woods et al., 2012). This might potentially be explained by differences in the baseline scores of cohorts in different studies; indeed, low neuropsychiatric symptom scores and high quality of life scores at baseline would make it difficult to demonstrate improvements. This notion was consistent with results from a randomized controlled study by Clare et al. (2019), who also explained the lack of findings by low baseline scores. In addition, Clare et al. (2019) noted that the quantitative findings were at odds with the results from the qualitative analysis, which indicated that the intervention may have provided benefits that were not detected by available standardized measures. Moreover, the intervention might prevent symptoms, rather than cure them, which would be difficult to detect with available outcome measures (Mittelman, 2008).

When examining the caregivers, the present study revealed no significant changes between the control and intervention group in caregiver quality of life or anxiety and depression scales. This finding was not surprising, because the intervention primarily targeted the person with dementia, not the caregiver. This finding was consistent with findings in other studies that also found no effect on caregivers (Woods et al., 2012). For example, a large metareview by Orgeta et al. (2015) demonstrated that the psychological treatment had no effect on caregivers self-rated depressive symptoms. In contrast, a study by Teri et al. (1997) found that the depressive 
symptoms in caregivers decreased, even though the patient was the main target of behavioral treatment. One explanation might be the high proportion of clinical depression in the caregivers studied by Teri et al. (1997). In the present study, the mean score for caregivers was below the cutoff for depression at baseline (Bjelland et al., 2002). Therefore, there was little opportunity for the intervention to demonstrate improvements. The same limitation applied to the caregiver's burden, because the baseline scores were already at a floor level before treatment commenced. However, the caregivers might have experienced the intervention as positive and valuable, even though it could not be demonstrated quantitatively. Indeed, a qualitative analysis of the caregivers included in the present study, completed 6-12 months after the interventions, showed that they had gained knowledge of dementia and insight into the importance of focusing on pleasant activities (Myhre et al., 2018). Also, the caregivers referred to the intervention as a positive experience and it contributed to learning ways to cope with dementia. Hence, further qualitative research design might be able to pick up valuable nuances of caregiver improvements that are missed with the outcome measures used in this study.

\section{Strengths and limitations}

A common concern in longitudinal studies is postassignment attrition, which results in losing to follow-up the people with the worst illness and fragility (Salthouse, 2013). However, a major strength of the present study was that those that completed the study were not significantly different in baseline characteristics from those that discontinued the study. Other strengths were that the therapist self-ratings indicated good treatment adherence, the findings were recorded according to the CONSORT guidelines, and the planned sample size was achieved with adequate power.

Nevertheless, there were several limitations. The control and intervention groups had different baseline characteristics. However, we adjusted for virtually all the differences with the ANCOVA. Also, female participants were underrepresented in the present study. Therefore, stratification might have been a better approach. However, some of the participation centers only included a small number of participants; consequently, stratification would not have been possible within the available time frame and funding. In addition, the "mixed" sample of patients with amnestic MCI and dementia could be a limitation, because some patients with MCI might have been healthy older people; indeed, a previous study showed that $14-50 \%$ of people with MCIs reverted to normal function (Butters et al., 2008; Petersen et al., 2018). On the other hand, there were few baseline differences between the people with MCI and dementia in this study. The mixed sample might have represented a realistic target for outpatient healthcare settings, because in practice, the distinction between amnestic MCI and dementia often only depends on the premorbid cognitive level. Consequently, although the sample in this study was heterogeneous, we considered it representative of people with MCI and mild dementia due to Alzheimer's disease. In addition, another limitation may be the heterogeneity of the therapists. However, they all received regular supervision and workshops during the study. Also, the heterogeneity reflects the heterogeneity that is seen in real-life practice. Lastly, the multicomponent character of the intervention implied that the "active ingredients" of the psychosocial intervention are difficult to identify. On the other hand, it is just this feature that enables goal-oriented individual therapy.

\section{Future studies}

Future studies could apply a mixed-methods approach. A combination of qualitative and quantitative evaluations of an intervention might provide more insight into how people with dementia experience psychosocial interventions, which might generate ideas about how interventions work (Moniz-Cook et al., 2011). Also, future studies could provide qualitative interviews of the coping experience of psychosocial interventions in combination with measurement scales that grasp the positive aspects of psychosocial interventions, for example, the Coping Orientation to Problems Experienced Inventory (Carver et al., 1989) or the Client Satisfaction Instrument (Attkisson and Zwick, 1982). Also, more active control groups could be applied, such as comparing the CORDIAL intervention with another active treatment, for instance, cognitive stimulation therapy in groups.

Finally, future research should evaluate the longterm effects of psychosocial interventions, measured 2 to 3 years after the intervention. Those studies might reveal whether psychosocial interventions delivered in the early stages of the disease could be able to prevent the development of concurrent or recurrent depression.

\section{Conclusion}

In conclusion, the CORDIAL program, a multimodal intervention comprising 11 individual sessions comprising elements of CBT, cognitive rehabilitation, and reminiscence therapy for people 
with MCI and dementia, reduced depressive symptoms in individuals with MCIs and dementia.

\section{Conflicts of interest}

None.

\section{Description of authors' roles}

J.B. Tonga wrote the main parts of the manuscript, and I.D Ulstein, E. Arnevik, M. Korsnes, and $\mathrm{K}$. Werheid assisted with critical content in the manuscript. J. Satyte-Benth completed the statistical analysis and assisted with writing the results. J.B. Tonga and I.D. Ulstein recruited the participants and collected the data. K. Werheid developed the original German CORDIAL study, together with A.I. Thone-Otto. I.D. Ulstein developed the Norwegian CORDIAL study.

\section{Acknowledgments}

The CORDIAL study was funded by the Norwegian Health Association, the Old Age Psychiatry Research Network, Telemark Hospital Trust and Vestfold Trust (TeVe), the Department of Old Age Psychiatry, Oslo University Hospital, and the Civitan Norway Research Foundation of Alzheimer's Disease. The authors did not enter into an agreement with a funding organization.

The research team would like to thank all the participants and caregivers that participated in this study, and the health staff that helped with the study. Thanks to A.I. Thone-Otto, who conjointly developed the CORDIAL program with $\mathrm{K}$. Werheid. We would also like to thank the following hospitals for participating:

Department of Geriatric Medicine, Institute of Clinical Medicine, Oslo University Hospital, Ullevål, Norway; Department of Old Age Psychiatry, Oslo University Hospital, Grønland, Norway; Department of Old Age Psychiatry, Akershus University Hospital, Skytta, Norway; Department of Old Age Psychiatry, Innlandet Hospital Trust, Sanderud, Norway; and Department of Old Age Psychiatry, Vestfold Hospital Trust, Granli, Norway.

\section{Supplementary material}

To view supplementary material for this article, please visit https://doi.org/10.1017/S1041610220000216

\section{References}

Alzheimer's Association. (2014). 2014 Alzheimer's disease facts and figures. Alzheimer's $\mathcal{E}$ Dementia, 10, e47-e92.
Appelhof, B. et al. (2019). Effects of a multidisciplinary intervention on the presence of neuropsychiatric symptoms and psychotropic drug use in nursing home residents with young-onset dementia: Behavior and Evolution of Young ONset Dementia part 2 (BEYOND-II) study. The American fournal of Geriatric Psychiatry, 27, 581-589.

Attkisson, C. C. and Zwick, R. (1982). The client satisfaction questionnaire: psychometric properties and correlations with service utilization and psychotherapy outcome. Evaluation and Program Planning, 5, 233-237.

Banningh, L. W. J.-W., Prins, J. B., Vernooij-Dassen, M. J., Wijnen, H. H., Rikkert, M. G. O. and Kessels, R. P. (2011). Group therapy for patients with mild cognitive impairment and their significant others: results of a waiting-list controlled trial. Gerontology, 57, 444-454.

Beck, J. S. (2011). Cognitive Behavior Therapy: Basics and Beyond. New York: Guilford Press.

Bjelland, I., Dahl, A. A., Haug, T. T. and Neckelmann, D. (2002). The validity of the Hospital Anxiety and Depression Scale: an updated literature review. fournal of Psychosomatic Research, 52, 69-77.

Brewer, J. B., Magda, S., Airriess, C. and Smith, M. E. (2009). Fully-automated quantification of regional brain volumes for improved detection of focal atrophy in Alzheimer disease. American fournal of Neuroradiology, 30, 578-80.

Brodaty, H., Green, A. and Koschera, A. (2003). Metaanalysis of psychosocial interventions for caregivers of people with dementia. Fournal of the American Geriatrics Society, 51, 657-664.

Brueggen, K. et al. (2017). Cognitive rehabilitation in Alzheimer's disease: a controlled intervention trial. fournal of Alzheimer's Disease, 57, 1315-1324.

Brækhus, A., Ulstein, I., Wyller, T. and Engedal, K. (2011). The memory clinic - outpatient assessment when dementia is suspected. Tidsskrift for Norsk Legeforening, 22.

Butters, M. A. et al. (2008). Pathways linking late-life depression to persistent cognitive impairment and dementia. Dialogues in Clinical Neuroscience, 10, 345.

Carver, C. S., Scheier, M. F. and Weintraub, J. K. (1989). Assessing coping strategies: a theoretically based approach. fournal of Personality and Social Psychology, 56, 267.

Chatfield, M. D., Brayne, C. E. and Matthews, F. E. (2005). A systematic literature review of attrition between waves in longitudinal studies in the elderly shows a consistent pattern of dropout between differing studies. fournal of Clinical Epidemiology, 58, 13-19.

Clare, L. et al. (2019). Individual goal-oriented cognitive rehabilitation to improve everyday functioning for people with early-stage dementia: a multi-centre randomised controlled trial (the GREAT trial). International fournal of Geriatric Psychiatry, 34, 709-721.

Clare, L. et al. (2010). Goal-oriented cognitive rehabilitation for people with early-stage Alzheimer disease: a single-blind randomized controlled trial of clinical efficacy. The American fournal of Geriatric Psychiatry, 18, 928-939.

Cody, R. A. and Drysdale, K. (2013). The effects of psychotherapy on reducing depression in residential aged care: a meta-analytic review. Clinical Gerontologist, 36, 46-69.

Cotelli, M., Manenti, R. and Zanetti, O. (2012). Reminiscence therapy in dementia: a review. Maturitas, 72, 203-205. 
Dubois, B., Feldman, H. H., Jacova, C., Dekosky, S. T., Barberger-Gateau, P. and Cummings, J. (2007). Research criteria for the diagnosis of Alzheimer's disease: revising the NINCDS-ADRDA criteria. The Lancet Neurology, 6, 734-746.

Enache, D., Winblad, B. and Aarsland, D. (2011). Depression in dementia: epidemiology, mechanisms, and treatment. Current Opinion in Psychiatry, 24, 461-472.

Engedal, K., Brækhus, A., Andreassen, O. and Nakstad, P. (2012). Diagnosis of dementia -automatic quantification of brain structures. Tidsskrift for den Norske laegeforening: tidsskrift for praktisk medicin, ny raekke, 132, 1747-1751.

Fantino, B. and Moore, N. (2009). The self-reported Montegomery-Aasberg depression rating scale is a useful evaluative tool in major depressive disorder. $B M C$ Psychiatry, 9, 26.

Field, A. (2013). Discovering statistics using IBM SPSS statistics. London: Sage.

Folstein M. F., Folstein S. E. and McHugh P. R. (1975). "Mini-mental state": a practical method for grading the cognitive state of patients for the clinician. Fournal of Psychiatric Research, 12, 189-198.

Greene, J., Smith, R., Gardiner, M. and Timbury, G. (1982). Measuring behavioural disturbance of elderly demented patients in the community and its effects on relatives: a factor analytic study. Age and Ageing, 11, 121-126.

Harman, G. and Clare, L. (2006). Illness representations and lived experience in early-stage dementia. Qualitative Health Research, 16, 484-502.

Huang, H.-C. et al. (2015). Reminiscence therapy improves cognitive functions and reduces depressive symptoms in elderly people with dementia: a meta-analysis of randomized controlled trials. Fournal of the American Medical Directors Association, 16, 1087-1094.

Hyer, L., Yeager, C. A., Hilton, N. and Sacks, A. (2009). Group, individual, and staff therapy: an efficient and effective cognitive behavioral therapy in long-term care. American fournal of Alzheimer's Disease $\mathcal{E}$ Other Dementias ${ }^{\circledR}$, 23, 528-539.

Kaufer, D. I. et al. (2000). Validation of the NPI-Q, a brief clinical form of the Neuropsychiatric Inventory. The fournal of Neuropsychiatry and Clinical Neurosciences, 12, 233-239.

Kim, Y. O. and Shim, M. S. (2015). Cognitive functions, instrumental activities of daily living, depression and quality of life in the elderly with mild cognitive impairment. fournal of Korean Public Health Nursing, 29, 219-230.

Knapskog, A.-B., Barca, M. L., Engedal, K. and Group, C. S. (2011). A comparison of the validity of the Cornell Scale and the MADRS in detecting depression among memory clinic patients. Dementia and Geriatric Cognitive Disorders, 32, 287-294.

Kurz, A., Pohl, C., Ramsenthaler, M. and Sorg, C. (2009). Cognitive rehabilitation in patients with mild cognitive impairment. International fournal of Geriatric Psychiatry: A Fournal of the Psychiatry of Late Life and Allied Sciences, 24, 163-168.

Kurz, A. et al. (2012). CORDIAL: cognitive rehabilitation and cognitive-behavioral treatment for early dementia in Alzheimer disease a multicenter, randomized, controlled trial. Alzheimer Disease \& Associated Disorders, 26, 246-253.
Lawton, M. and Brody, E. (1969). Instrumental activites of daily living scale (IADL). The Gerontologist, 9, 179-186.

Lee, S. M., Roen, K. and Thornton, A. (2014). The psychological impact of a diagnosis of Alzheimer's disease. Dementia, 13, 289-305.

Logsdon, R. G., Gibbons, L. E., Mccurry, S. M. and Teri, L. (1999). Quality of life in Alzheimer's disease: patient and caregiver reports. Fournal of Mental Health and Aging, 5, 21-32.

Mittelman, M. (2008). Psychosocial intervention research: challenges, strategies and measurement issues. Aging $\mathcal{E}$ Mental Health, 12, 1-4.

Mittmann, N., Mitter, S., Borden, E. K., Herrmann, N., Naranjo, C. A. and Shear, N. H. (1997). MontgomeryÅsberg severity gradations. The American fournal of Psychiatry, 154, 1320-1321.

Moniz-Cook, E., Vernooij-Dassen, M., Woods, B., Orrell, M. and Network, I. (2011). Psychosocial interventions in dementia care research: the INTERDEM manifesto. Aging \& Mental Health, 15, 283-290.

Montgomery, S. A. and Åsberg, M. (1979). A new depression scale designed to be sensitive to change. The British fournal of Psychiatry, 134, 382-389.

Myhre, J., Tonga, J. B., Ulstein, I. D., Høye, S. and Kvaal, K. (2018). The coping experiences of spouses of persons with dementia. Fournal of Clinical Nursing, 27, e495-e502.

Müller-Thomsen, T., Arlt, S., Mann, U., Maß, R. and Ganzer, S. (2005). Detecting depression in Alzheimer's disease: evaluation of four different scales. Archives of Clinical Neuropsychology, 20, 271-276.

Müller, M. J., Szegedi, A., Wetzel, H. and Benkert, O. (2000). Moderate and severe depression: gradations for the Montgomery-Åsberg depression rating scale. fournal of Affective Disorders, 60, 137-140.

Olazarán, J. et al. (2010). Nonpharmacological therapies in Alzheimer's disease: a systematic review of efficacy. Dementia and Geriatric Cognitive Disorders, 30, 161-178.

Orgeta, V., Qazi, A., Spector, A. and Orrell, M. (2015). Psychological treatments for depression and anxiety in dementia and mild cognitive impairment: systematic review and meta-analysis. The British fournal of Psychiatry, 207, 293-298.

Petersen, R. C. et al. (2018). Practice guideline update summary: mild cognitive impairment: report of the guideline development, dissemination, and implementation subcommittee of the American academy of neurology. Neurology, 90, 126-135.

Prado-Jean, A. et al. (2010). Specific psychological and behavioral symptoms of depression in patients with dementia. International fournal of Geriatric Psychiatry, 25, 1065-1072.

Salthouse, T. A. (2013). Selectivity of attrition in longitudinal studies of cognitive functioning. Fournals of Gerontology Series B: Psychological Sciences and Social Sciences, 69, 567-574.

Scheltens, P. et al. (1992). Atrophy of medial temporal lobes on MRI in" probable" Alzheimer's disease and normal ageing: diagnostic value and neuropsychological correlates. Fournal of Neurology, Neurosurgery \& Psychiatry, 55, 967-972. 
Schwarzer, R., BÄßler, J., Kwiatek, P., Schröder, K. and Zhang, J. X. (1997). The assessment of optimistic selfbeliefs: comparison of the German, Spanish, and Chinese versions of the general self-efficacy scale. Applied Psychology, $46,69-88$.

Sikkes, S., de Lange-de Klerk, E., Pijnenburg, Y. and Scheltens, P. (2009). A systematic review of instrumental activities of daily living scales in dementia: a room for improvement. Fournal of Neurology, Neurosurgery $\mathcal{E}$ Psychiatry, 80, 7-12.

Selbaek, G., Kirkevold, Ø., Sommer, O. H. and Engedal, K. (2008). The reliability and validity of the Norwegian version of the Neuropsychiatric Inventory, nursing home version (NPI-NH). International Psychogeriatrics, 20, 375-382.

Snowden, B. et al. (2015). Longitudinal association of dementia and depression. American fournal of Geriatric Psychiatry, 23, 897-905.

Stockwell-Smith, G., Moyle, W. and Kellett, U. (2018). The impact of early psychosocial intervention on selfefficacy of care recipient/carer dyads living with early-stage dementia-A mixed-methods study. Fournal of Advanced Nursing, 74, 2167-2180.

Strobel, C. and Engedal, K. (2008). MMSE-NR. Norsk revidert Mini Mental Status Evaluering. Revidert og utvidet manual. Tønsberg: Nasjonalt kompetansesenter for aldring og helse.

Teri, L., Logsdon, R. G., Uomoto, J. and Mccurry, S. M. (1997). Behavioral treatment of depression in dementia patients: a controlled clinical trial. The fournals of Gerontology Series B: Psychological Sciences and Social Sciences, 52, P159-P166.

Teri, L., Mckenzie, G. and Lafazia, D. (2005). Psychosocial treatment of depression in older adults with dementia. Clinical Psychology: Science and Practice, 12, 303-316.

Tonga, J. B., Karlsoeen, B. B., Arnevik, E. A., Werheid, K., Korsnes, M. S. and Ulstein, I. D. (2016). Challenges with manual-based multimodal psychotherapy for people with Alzheimer's disease: a case study. American fournal of Alzheimer's Disease $\mathcal{E}$ Other Dementias, 31, 311-317.
Tonga, J. B., Arnevik, E. A., Werheid, K. and Ulstein, I. D. (2015). Manual-based cognitive behavioral and cognitive rehabilitation therapy for young-onset dementia: a case report. International Psychogeriatrics, 28, 119-122.

Thorgrimson, L. et al. (2003). Whose quality of life is it anyway? The validity and reliability of the Quality of LifeAlzheimer's Disease (QoL-AD) scale. Alzheimer Disease $\mathcal{E}$ Associated Disorders, 17, 201-208.

Ulstein, I., Bruun Wyller, T. and Engedal, K. (2007). The relative stress scale, a useful instrument to identify various aspects of carer burden in dementia? International fournal of Geriatric Psychiatry: A fournal of the Psychiatry of Late Life and Allied Sciences, 22, 61-67.

Wang, J. J. (2007). Group reminiscence therapy for cognitive and affective function of demented elderly in Taiwan. International fournal of Geriatric Psychiatry: $A$ Fournal of the Psychiatry of Late Life and Allied Sciences, 22, 1235-1240.

Wimo, A. W. and Winblad, B. (2003). Resource utlization in dementia: RUD Lite. Brain Aging, 3, 48-59.

Winblad, B. et al. (2004). Mild cognitive impairment beyond controversies, towards a consensus: Report of the international working group on mild cognitive impairment. Fournal of Internal Medicine, 256, 240-246.

Werheid, K., Köhncke, Y., Ziegler, M. and Kurz, A. (2015). Latent change score modeling as a method for analyzing the antidepressant effect of a psychosocial intervention in Alzheimer's disease. Psychotherapy and Psychosomatics, 84, 159-166.

Woods, R. T. et al. (2012). REMCARE: reminiscence groups for people with dementia and their family caregivers-effectiveness and cost-effectiveness pragmatic multicentre randomised trial. Health Technology Assessment, 16, 1-16.

Woods, B., O'Philbin, L., Farrell, E. M., Spector, A. E. and Orrell, M. (2018). Reminiscence therapy for dementia. Cochrane Database of Systematic Reviews, Art. No. CD001120.

Zigmond, A. S. and Snaith, R. P. (1983). The hospital anxiety and depression scale. Acta Psychiatrica Scandinavica, $67,361-370$. 